\title{
Role of miRNA-324-5p-Modified Adipose-Derived Stem Cells in Post-Myocardial Infarction Repair
}

\author{
Zhou Ji ${ }^{1}$, Chan Wang ${ }^{2}$, Qing Tong ${ }^{3}$ \\ ${ }^{I}$ Department of Cardiovascular Medicine, The Third Affiliated Hospital of Finzhou Medical University, Finzhou, China \\ 'Finzhou Hospital of Traditional Chinese Medicine, Finzhou, China \\ ${ }^{3}$ Office of Academic Research, The Third Affiliated Hospital of Finzhou Medical University, Finzhou, China
}

Background and Objectives: To seek out the role of mircoRNA (miR)-324-5p-modified adipose-derived stem cells (ADSCs) in post-myocardial infarction (MI) myocardial repair.

Methods and Results: Rat ADSCs were cultivated and then identified by morphologic observation, osteogenesis and adipogenesis induction assays and flow cytometry. Afterwards, ADSCs were modified by miR-324-5p lentiviral vector, with ADSC proliferation and migration measured. Then, rat MI model was established, which was treated by ADSCs or miR-324-5p-modified ADSCs. Subsequently, the function of miR-324-5p-modified ADSCs in myocardial repair of MI rats was assessed through functional assays. Next, the binding relation of miR-324-5p and Toll-interacting protein (TOLLIP) was validated. Eventually, functional rescue assay of TOLLIP was performed to verify the role of TOLLIP in MI. First, rat ADSCs were harvested. Overexpressed miR-324-5p improved ADSC viability. ADSC transplantation moderately enhanced cardiac function of MI rats, reduced enzyme levels and decreased infarct size and apoptosis; while miR-324-5p-modified ADSCs could better promote post-MI repair. Mechanically, miR-324-5p targeted TOLLIP in myocardial tissues. Moreover, TOLLIP overexpression debilitated the promotive role of miR-324-5p-modified ADSCs in post-MI repair in rats.

Conclusions: miR-324-5p-modified ADSCs evidently strengthened post-MI myocardial repair by targeting TOLLIP in myocardial tissues.

Keywords: Myocardial infarction, Radiotherapy, Adipose-derived stem cells, mircoRNA-324-5p, Myocardial repair, Stem cell survival

Received: February 3, 2021, Revised: April 26, 2021,

Accepted: April 30, 2021, Published online: June 30, 2021

Correspondence to Zhou Ji

Department of Cardiovascular Medicine, The Third Affiliated

Hospital of Jinzhou Medical University, No. 2, section 5, Heping

Road, Linghe District, Jinzhou 121000, China

Tel: +86-0416-3999186, Fax: +86-0416-3999052

E-mail: jizhou1214@163.com

(c) This is an open-access article distributed under the terms of the Creative Commons Attribution Non-Commercial License (http://creativecommons.org/ licenses/by-nc/4.0/), which permits unrestricted non-commercial use, distribution, and reproduction in any medium, provided the original work is properly cited.

Copyright (c) 2021 by the Korean Society for Stem Cell Research

\section{Introduction}

Myocardial infarction (MI) emerges as a major cause of physical disability and even mortality since it induces clinical manifestation like vomiting, continuous chest pain, nausea, depression, fatigue, feebleness, arrhythmias and tachypnea $(1,2)$. MI is strongly associated with the perilous factors embracing high lipid concentration, diabetes or hypertension, lack of physical exercise, unbalanced diet, overweight and smoking, which aggregate intravascular clots, eliminate oxygenation, disrupt vascular endothelium construction and aggravate atherosclerosis in MI patients (3). Furthermore, MI can trigger undesirable consequences, for example, myocardial fail- 
ure, high rate of relapse and mortality (4). To avoid those poor prognostic responses, candidate treatment that targets MI severity is urgently required (1).

Hence, treatment of cells, especially mesenchymal stem cells (MSCs) attracts increasing attention in myocardial repair by their high differentiation and self-renewal ability and features of angiogenesis and immunomodulation (5). Besides, MSCs could practically strengthen heart function, decrease scar range and promote myocardial repair through suppressing cardiac fibrosis, mediating myocardial cellular behaviors and modulating immune responses (6). Significantly, when adipose-derived SCs (ADSCs) are introduced into MI area, myocardial self-renewal and repair are promoted (7). Moreover, compared with the other MSCs, ADSCs could better reduce MI range and scar size (8). However, although the administration of MSC contributes a lot to heart diseases, the survival rate of these cells is still a disappointment (9). In other word, to facilitate viability and migration of ADSCs might be a breakthrough in post-MI alleviation and repair.

A number of studies have suggested that the modification of microRNAs (miRs) to MSCs prevents cells from death or autophagy, restricting MI size and improving myocardial repair of MI tissues $(10,11)$. miRs are deemed as reliable biomarkers of $\mathrm{MI}$ as they regulate downstream genes or axis and affect myocardial function (12). miR-324 is downregulated in cardiomyocytes with hypoxia/reoxygenation-induced injury, inhibiting cell proliferation (13). However, research about the specific function of miR-324-5p in MI progression is elusive. Here, we found that miR-324-5p could promote the viability and motility of ADSCs, which is corroborated by a recent study that miR-324-5p encourages adipocyte differentiation and adipogenic level (14). What's more, Chen et al. (15) have revealed that miR-324-5p protect myocardial tissues against MI injury by reducing mitochondrial fracture and oxidative stress damage, and enhancing endothelial progenitor cell viability. Besides, as a target gene of miR-324-5p, Toll-interacting protein (TOLLIP) is overexpressed in MI tissues and it degenerates inflammatory reaction of MI and spoils cardiac function (16). By means of these information, we hypothesize that miR-324-5pmodified ADSCs could greatly improve post-MI myocardial repair by targeting TOLLIP.

\section{Materials and Methods}

\section{Experimental animal}

Male Sprague-Dawley (SD) rats (4 weeks, 100 110 g)
(Beijing Vital River Laboratory Animal Technology Co., Ltd, Beijing, China, SYXK (Beijing) 2017-0033) were kept in a controlled environment with constant humidity $(60 \% \pm 5 \%)$, and temperature $\left(22 \pm 3^{\circ} \mathrm{C}\right)$ for $12 \mathrm{~h}$ light/dark cycles with free access to food and water. All the operations were committed under anesthesia.

\section{Separation and cultivation of ADSCs}

ADSCs were separated and cultivated as previously reported (17). Briefly, inguinal adipose tissues of SD rats were separated and cleaned with phosphate buffer saline (PBS) to discard the residual blood. Then, tissues were cut into pieces $(1 \times 1 \mathrm{~mm})$ which were detached with collagenase I. After the centrifugation at 4,000 g for $5 \mathrm{~min}$, cell particles were suspended in Dulbecco's modified Eagle's medium (DMEM) consisting of 10\% fetal bovine serum (FBS) $/ 1 \%$ penicillin-streptomycin/2 $\mathrm{mM} \mathrm{L-glutamine} \mathrm{at}$ $37^{\circ} \mathrm{C}$ for $48 \mathrm{~h}$ in a humidified atmosphere with $5 \% \mathrm{CO}_{2}$. The medium consisting of non-adherent cells was refreshed by new medium every 3 days. The morphology of ADSCs was observed under a microscope. Cells were subcultured when they reached $90 \%$ confluence and experiments were performed using passaged 3 (P3) ADSCs.

\section{Identification of ADSCs}

Immunophenotyping of ADSCs was analyzed on a FACScalibur flow cytometer (BectonDickinson, Bedford, MA, USA) with CD90 (ab225), CD45 (ab6329), CD105 (ab2529), CD34 (ab81289) antibodies (all from Abcam Inc., Cambridge, MA, USA).

Subsequently, adipogenic and osteogenic differentiation assays were committed. Osteogenic differentiation went as: ADSCs at $80 \sim 90 \%$ confluence were cultured in osteogenic induction medium, which included DMEM containing 10\% FBS, ascorbic acid, $\beta$-glycerophosphate, and dexamethasone, from which ADSCs were cultured to induce osteogenic differentiation. Adipogenic differentiation was conducted as ADSCs were incubated in adipogenic induction medium, which included DMEM containing 10\% FBS, insulin, indomethacin, dexamethasone, and 3-isobutyl-1-methylxanthine, from which ADSCs were cultivated to induce adipogenic differentiation. The medium was refreshed every 3 4 days for 21 days. Subsequently, cells were fixed and stained with alizarin red or oil red O. All reagents were attained from Beijing Solarbio Science \& Technology Co., Ltd. (Beijing, China).

\section{Treatment of ADSCs}

P3 ADSCs were incubated by DMEM with $10 \%$ FBS in the 6-well plates till $70 \%$ confluence. At this point, 
ADSCs were divided into the blank group (uninfected), the lentiviral vector (LV, Shanghai GenePharma Co, Ltd, Shanghai, China)-negative control (NC) group (infected with NC), and the LV-miR group (infected with miR324-5p overexpression lentivirus). Then, the LV-NC group or the LV-miR-324-5p group was infected with $8 \mu \mathrm{g} / \mathrm{ml}$ polybrene (Solarbio) into ADSCs (18-20).

\section{Cell counting kit-8 (CCK-8) method}

Cells $\left(5 \times 10^{3}\right.$ cells/well) with various treatment were inoculated into 96 -well plates at $37^{\circ} \mathrm{C}$ for $24 \mathrm{~h}, 48 \mathrm{~h}$ and $72 \mathrm{~h}$, respectively, with $10 \mu \mathrm{l}$ CCK-8 reagent (Shanghai Beyotime Biotechnology Co. Ltd., Shanghai, China) supplemented into each well. Next, optical density at $450 \mathrm{~nm}$ was assessed using a microplate reader (Bio-Rad, Inc., Hercules, CA, USA).

\section{Transwell assay}

ADSCs were detached by trypsin, centrifuged and soaked twice in PBS. Afterwards, ADSCs were resuspended in DMEM consisting of 10\% FBS. Then, $100 \mu 1$ cell suspension $\left(1 \times 10^{4}\right.$ cells $)$ and $100 \mu 1$ DMEM consisting of $10 \%$ FBS were supplemented into the 24-well plates, which were then placed in Transwell chamber and cultivated at $37^{\circ} \mathrm{C}$ for $24 \mathrm{~h}$. The Transwell chamber was subsequently removed, and the cells were immersed into PBS, fixed in 4\% formic acid (Solarbio) for $10 \mathrm{~min}$, and then stained with Hoechst 33258 ( $1 \mu \mathrm{g} / \mathrm{ml}$, Solarbio) for $30 \mathrm{~min}$ in the dark. Finally, cells were rinsed 3 times in PBS, with the total number of migrated cells counted by a microscope.

\section{Establishment and treatment of $\mathrm{MI}$ rat model}

Rats were anesthetized with pentobarbital sodium (30 $\mathrm{mg} / \mathrm{kg}$, Sigma-Aldrich, Merck KGaA, Darmstadt, Germany) via intraperitoneal injection using a ventilator connected to trachea cannula. Heart was exposed in the fourth and fifth intercostal gap after left thoracotomy, when left anterior descending coronary artery (LAD) running in myocardial tissues consistent with the direction of the great myocardial vein was found, it was sutured and ligated with 6-0 silk thread. After LAD occlusion, the corresponding blood supply area was whitened, while infarction manifestations such as ST segment elevation was noticed as electrocardiogram displayed (11, 18, 20-23). Subsequent treatments were performed $30 \mathrm{~min}$ after ligation (22). Peri-MI areas were injected at multiple sites by PBS or $20 \mu 1$ PBS containing $1 \times 10^{6}$ differently treated ADSCs or adenovirus (Ad)-NC (empty vector $\mathrm{Ad}, 0.5 \times 10^{9} \mathrm{pfu}$ ) or Ad-TOLLIP (TOLLIP Ad, $0.5 \times 10^{9} \mathrm{pfu}$ ) (Ad-NC and
Ad-TOLLIP were both synthesized by Xinji Biotechnology Co., Ltd, Shanghai, China). The sham group received only thoracotomy without LAD. After ADSCs transplantation, all rats underwent echocardiography on the 28th day, and blood samples were collected, with 6 were used for reverse transcription quantitative polymerase chain reaction (RT-qPCR) and the remaining ones for hematoxylin and eosin (HE) staining and terminal deoxynucleotidyl transferase-mediated dUTP nick end labeling (TUNEL) staining.

\section{Myocardial function assessment}

Myocardial function of rats was evaluated using the Vevo2100 small animal ultrasound imaging system (VisualSonic, Toronto, Canada). In brief, rats were anesthetized with $40 \%$ oxygen (GH-reagent, Shanghai, China) and isoflurane (1.7\%, final concentration), and an ultrasound probe was inserted into the short axis of the left chest to collect two dimensional echocardiogram of the papillary muscles. The left ventricular end-diastolic diameter (LVEDD) and LV end-systolic diameter (LVESD) were measured according to the myocardial contractility parameters $(18,20,24)$. LV ejection fraction was calculated as $\left((\mathrm{LVEDD})^{3}-\left(\mathrm{LVESD}^{3}\right) /\left(\mathrm{LVEDD}^{3}\right) \times 100 \%\right)$ and LV fractional shortening equals to ((LVEDD-LVESD)/ LVEDD) $\times 100 \%)(25)$.

\section{Myocardial enzyme assessment}

After the termination of echocardiography, orbital blood (1 ml) was taken, placed for $30 \mathrm{~min}$ and centrifuged at $3,000 \mathrm{~g}$ for $10 \mathrm{~min}$ at $4^{\circ} \mathrm{C}(26,27)$. Levels of myocardial troponin $\mathrm{T}$ (cTnT) (H149-4) and creatinine Kinase-MB (CK-MB) (H197) in serum were tested using the detection kits (NanJing JianCheng Bioengineering Institute, Nanjing, Jiangsu, China).

\section{HE staining}

The obtained myocardial tissues were paraffin-embedded and sliced at equal distances into sections $(6 \mu \mathrm{m})$ for the following HE staining. Specifically, sections were deparaffinized through biological transparent reagent (Solarbio) and hydrated with graded ethanol double distilled water, stained with hematoxylin (Beyotime) for 5 min, rinsed using double distilled water, differentiated with $1 \%$ hydrochloric acid alcohol for $30 \mathrm{~s}$ and then subjected to another double distilled water wash, followed by eosin (Beyotime) staining for $3 \mathrm{~min}$, successively dehydrated and transparent with graded ethanol absolute ethyl alcohol and biological transparent reagent $(14,28)$. In addition, sections were sealed and scanned with the digital 
scanning microscopy imaging system (Meyer Instruments Inc., Houston, TX, USA) to collect images.

\section{TUNEL staining}

Myocardial apoptosis was detected as myocardial tissue sections were assessed via the in situ cell death assay kit (Roche Applied Science, Mannheim GmbH, Penzberg, Germany) TUNEL staining. Next, the apoptotic nucleus and myocardial nucleus were stained with TUNEL or 4', 6-diamidino-2-phenylindole. Apoptotic rate was calculated as number of TUNEL-positive apoptotic myocardial cells/ total number of myocardial cells $\times 100 \%(21,28,29)$.

\section{Dual-luciferase reporter gene assay}

Luciferase reporter plasmids (pis CHECK2) containing TOLLIP wild type (WT) 3'untranslated regions (3'-UTRs) and TOLLIP mutant type (MUT) 3'-UTRs, respectively, were produced by General Biological System Co., Ltd. (Anhui, China). Lipofectamine 2000 (Invitrogen Inc., Carlsbad, CA, USA) was employed to co-transfect the reporter plasmids and miR-324-5p mimic or mimic $\mathrm{NC}$ (Shanghai Genechem Co., Ltd., Shanghai, China) to HEK293T cells (American Type Culture Collection, Manassas, Virginia, USA), which were inoculated into the 48-well plates. Then, luciferase activity of cell lysis buffer was examined through the Dual-Glo luciferase assay system (Promega Corporation, Madison, WI, USA).

\section{RT-qPCR}

The total RNA in ADSCs or myocardial tissues was extracted in line with the instructions of the TRIzol kits (Invitrogen). The total RNA concentration was determined using a spectrophotometer (German Eppendorf) and the purity of RNA was determined using 1\% agarose gel electrophoresis. Then, the RNA was adversely transcribed into cDNA with the PrimeScript RT kits (Takara Biotechnology Ltd., Dalian, China). The reverse transcription of miRNA was carried out by miRcute enhanced miRNA cDNA first strand synthesis kit (Beijing TianGen Biotech Co., Ltd. Beijing, China). RT-qPCR was performed when SYBR premix (Takara, Kusatsu, Japan) or miRcute enhanced miRNA fluorescence quantitative detection kit (SYBR Green) (TianGen Biotech) was applied on the Applied Biosystems 7500 PCR system (Carlsbad, CA, USA) following manufacturers' instructions. The $2^{-\Delta \Delta \mathrm{Ct}}$ method (30) was appointed to calculate the relative expression of genes with glyceraldehyde-3-phosphate dehydrogenase (GAPDH) as the internal reference for TOLLIP and U6 as the internal reference for miR-324-5p. $\triangle \triangle \mathrm{CT}=(\mathrm{CT}$ value of target gene in experimental group-
CT value of internal reference gene in experimental group) - (CT value of target gene in control group-CT value of internal reference gene in control group). Primer sequences of RT-qPCR were shown on Table 1.

\section{Statistical analysis}

SPSS 21.0 (IBM Corp. Armonk, NY, USA) was employed for data analysis and GraphPad Prism8.0 (GraphPad Software Inc., San Diego, CA, USA) for graph. Kolmogorov-Smirnov test indicated whether the data were in normal distribution. The results were displayed as mean \pm standard deviation. One-way analysis of variance (ANOVA) or two-way ANOVA for comparing each group, and Sidak's multiple comparisons test or Tukey's multiple comparisons test for pairwise comparisons after ANOVA. The $\mathrm{p}$ value was attained using a two-tailed test and $\mathrm{p}$ $<0.01$ indicated significant difference.

\section{Results}

\section{Cultivation and identification of rat ADSCs}

MI was a disease triggered by abrupt occlusion of coronary arteries and myocardial necrosis induced by ischemia and hypoxia $(31,32)$. Among various sources of SCs, MSCs, especially ADSCs, have been reported for the treatment of MI $(7,8)$. Although MSCs transplantation has witnessed a lot of successes in animal treatment, studies have shown that the cell survival rate after one week of transplantation was less than $10 \%(9,33,34)$. With the purpose of researching the role of ADSCs in MI, ADSCs were cultured, which exhibited the shape of long spindle under microscope (Fig. 1A). Induction differentiation carried out on ADSCs uncovered that cells were potent in getting adipogenesis and osteogenesis (Fig. 1B and 1C). Besides, CD105 (95.74\%) and CD90 (90.56\%), surface

Table 1. Primer sequences of RT-qPCR

\begin{tabular}{ll}
\hline \multicolumn{1}{c}{ Primer } & \multicolumn{1}{c}{ Sequences $\left(5^{\prime}-3^{\prime}\right)$} \\
\hline miR-324-5p & F: ATTGTACGCATCCCCTAGGGC \\
& R: CTCAACTGGTGTCGTGGA \\
TOLLIP & F: ATGGCGACCACCGTCAGCACGCA \\
& R: CTAGGACTCTTCGCCCATTGCAG \\
U6 & F: ATGGCGGACGACGTAGATCAGCA \\
RAPDH & F: ATGAGCCAACTCTCAATGGAGGGGC \\
& R: TTACTCCTTGGAGGCCATGTAGGC \\
\hline
\end{tabular}

Note: RT-qPCR: reverse transcription-quantitative polymerase chain reaction, miR: microRNA, TOLLIP: Toll-interacting protein, GAPDH: glyceraldehyde-3-phosphate dehydrogenase, F: forward, $\mathrm{R}$ : reverse. 
A

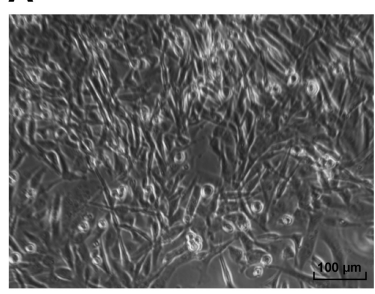

B

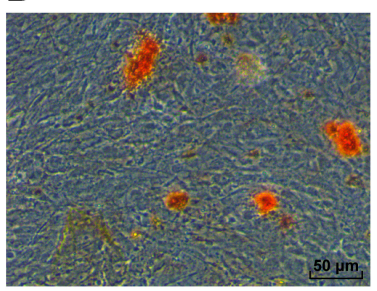

C
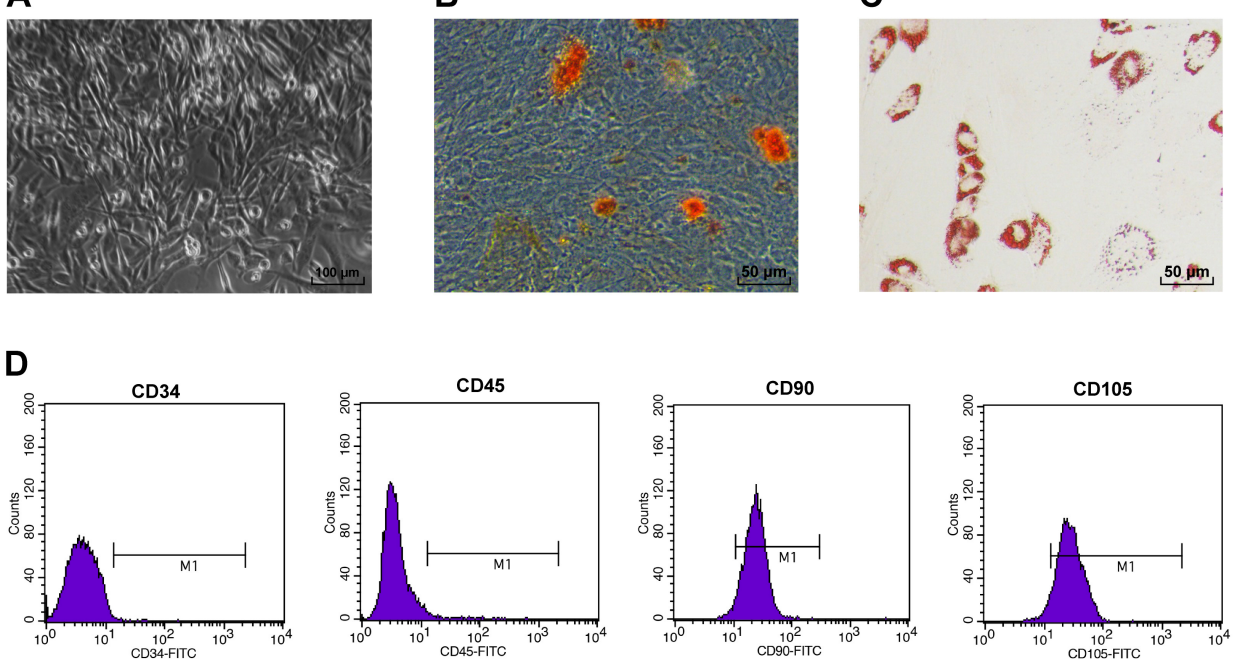

CD45

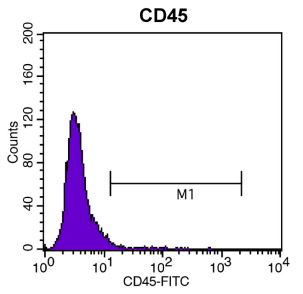

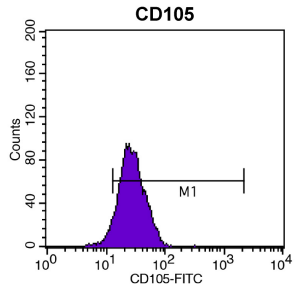

Fig. 1. Identification of rat ADSCs. Rat ADSCs were cultivated. Note: A, morphology of ADSCs observed under an inverted microscope. $B$ and $\mathrm{C}$, alizarin red (B) and oil red $\mathrm{O}(\mathrm{C})$ staining of ADSCs after adipogenesis and osteogenesis induction. D, expressions of CD34, CD45, CD105 and CD90 on the surface of ADSCs were detected by flow cytometry. The cell experiment was repeated three times.

A

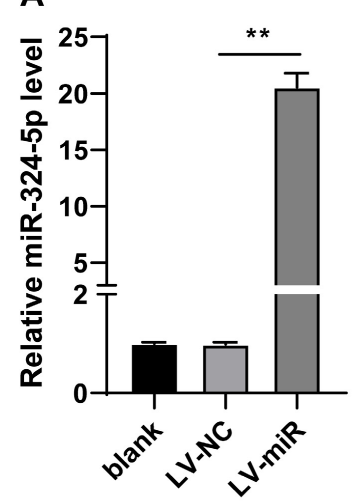

C

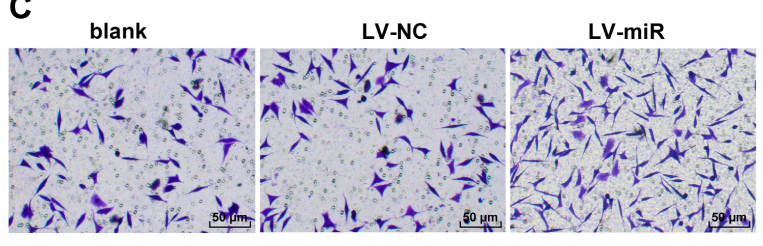

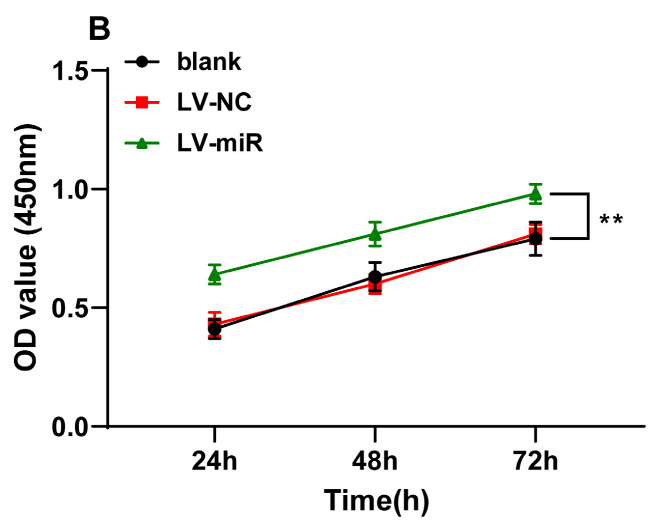

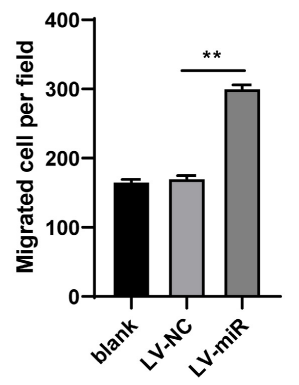

Fig. 2. miR-324-5p promotes ADSC proliferation and migration. Note: ADSCs were injected with LV-miR324-5p, with LV-NC as control. A, miR-324-5p expression in ADSCs was determined via RT-qPCR (A). B and $\mathrm{C}$, ADSC proliferation and migration were examined by CCK-8 method (B) and Transwell assay (C). The cell experiment was repeated three times; the results were shown as mean \pm standard deviation. Oneway ANOVA was used to analyze data in panels $A$ and $C$, and twoway ANOVA was used to analyze data in panel B. Tukey's multiple comparisons test was applied for post hoc test. ${ }^{* *} \mathrm{p}<0.01$. markers of MSCs, were overexpressed in ADSCs, while CD34 (1.21\%) and CD45 (2.38\%), markers of hematopoietic SCs, were not expressed (Fig. 1D). The above findings revealed that rat ADSCs were successfully cultured.

\section{miR-324-5p promotes ADSC proliferation and migration}

To explore the role of miR-324-5p on ADSCs, miR-324$5 \mathrm{p}$ expression was upregulated in ADSCs by the infection of ADSCs using LV-miR-324-5p ( $p<0.01$, Fig. 2A). It was unmasked from CCK-8 method and Transwell assay that ADSC proliferation and migration were enhanced in the LV-miR group compared with the LV-NC group $(\mathrm{p}<0.01$, Fig. $2 \mathrm{~B}$ and $2 \mathrm{C}$ ), indicating that overexpressed miR-324-5p enhanced ADSC dissemination.

\section{miR-324-5p-modified ADSCs enhance post-MI repair in rats}

To test the function of miR-324-5p-modified ADSCs in 
MI treatment, MI model was established in rats, with ADSCs administrated into the peri-MI area at multiple sites. As shown in Fig. 3A, myocardial function of MI rats was evidently impaired, while ADSCs treatment alleviated myocardial function impairment and decreased the serum levels of cTnT and CK-MB in MI rats, but the function of miR-ADSCs was even more efficient $(p<0.01$, Fig. 3A and $3 \mathrm{~B})$. HE staining and TUNEL staining conduced on myocardial tissue sections displayed that the degree of $\mathrm{MI}$ was aggravated and apoptosis was increased in MI rats, which was reversed in the ADSC group and the miR-ADSC group, and the miR-ADSC group exhibited was more effective than the ADSC group $(p<0.01$, Fig. $3 \mathrm{C}$ and $3 \mathrm{D})$. In summary, miR-324-5p overexpression in
ADSCs could improve post-MI myocardial repair in rats.

\section{miR-324-5p targets TOLLIP expression in MI rats}

Next, to further reveal the downstream mechanism of miR-324-5p in myocardial tissues, the downstream target genes of miR-324-5p was predicted and intersected by miRDB and Targetscan databases, and 16 target genes were noticed (Fig. 4A), of which TOLLIP was reported to promote the occurrence of MI (16). Thus, dual-luciferase reporter gene assay was designed based on the binding site of miR-324-5p and TOLLIP (Fig. 4B), which showed the target binding relationship between $\operatorname{miR}-324-5 \mathrm{p}$ and TOLLIP ( $\mathrm{p}<0.01$, Fig. $4 \mathrm{C})$. In addition, ADSCs treatment upregulated miR-324-5p expression and down-
A
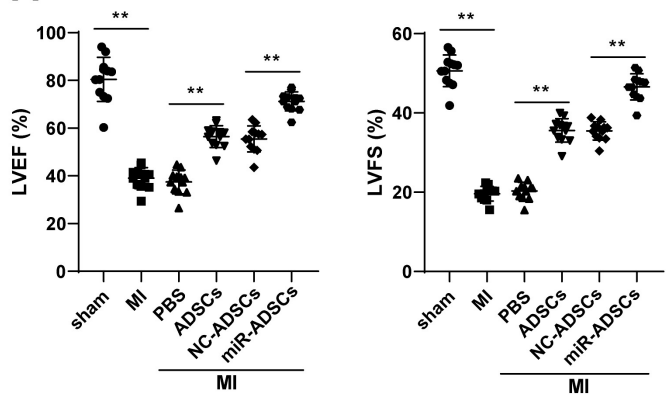

\section{B}
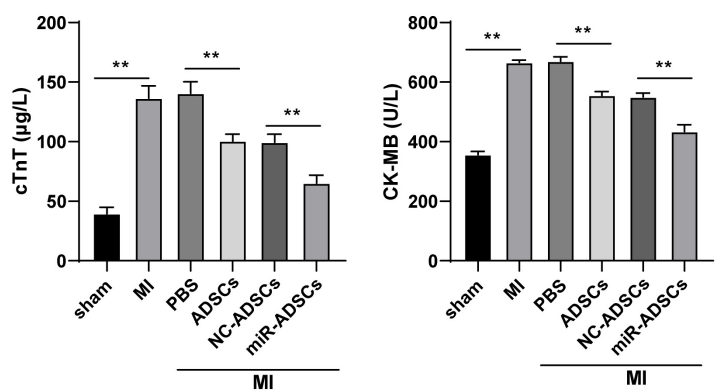
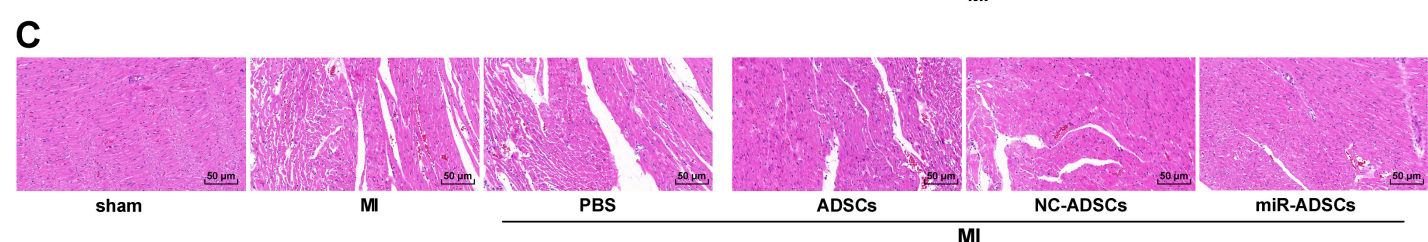

D
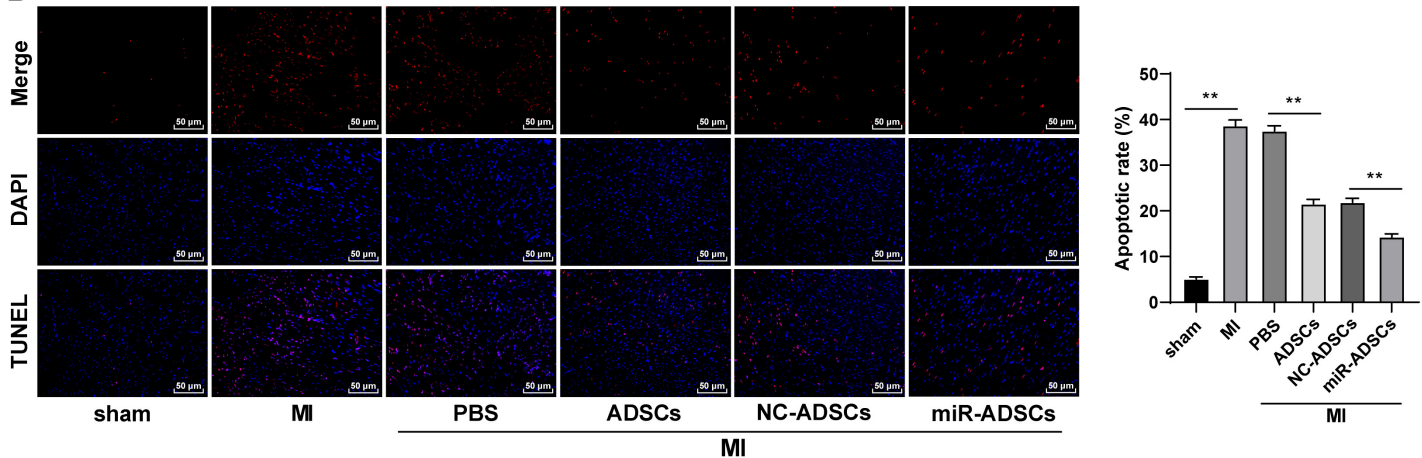

Fig. 3. miR-324-5p-modified ADSCs enhance post-Ml repair in rats. Note: Ml model was established in rats via LAD, with ADSCs or miR-324-5p-modified ADSCs injected into the peri-Ml area at multiple sites, with PBS injection as control. A, 28 days after ADSCs transplantation, myocardial function of rats was tested using echocardiography, $\mathrm{N}=12$. B, after ultrasound detections, levels of cTnT and CK-MB in serum of rats were measured, $\mathrm{N}=12$. $\mathrm{C}, \mathrm{Ml}$ degree in rats observed by HE staining. $\mathrm{D}$, apoptosis in $\mathrm{Ml}$ rats verified through TUNEL staining. $N=6$, the results of panels $B$ and $D$ were shown as mean \pm standard deviation. One-way ANOVA was used to analyze data in panels A, B and D. Tukey's multiple comparisons test was applied for post hoc test. ${ }^{* *} p<0.01$. MI: myocardial infarction, NC-ADSCs: ADSCs after lentivirus infection with miR-324-5p negative control, miR-ADSCs: ADSCs after lentivirus infection with miR-324-5p overexpression vectors. 


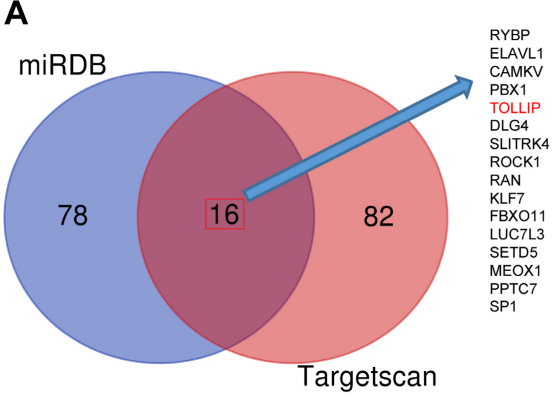

B

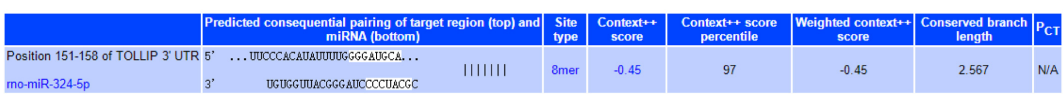

D

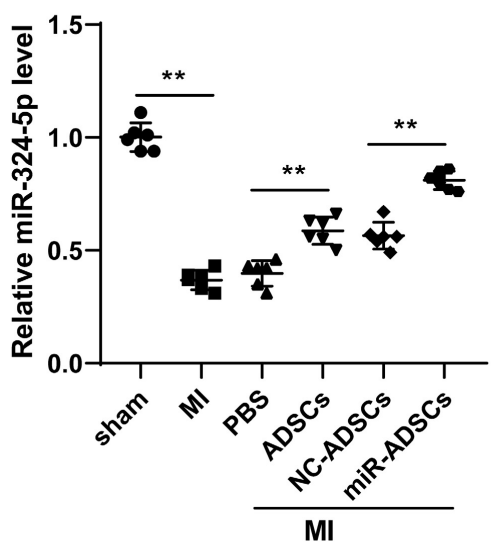

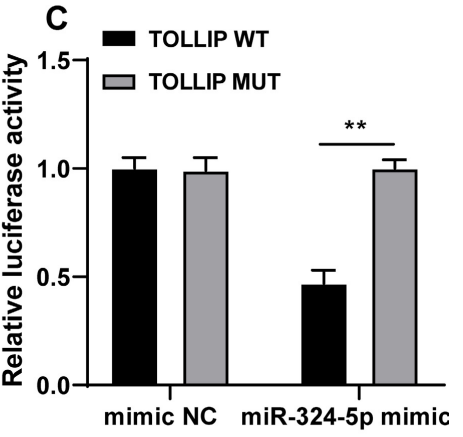

E

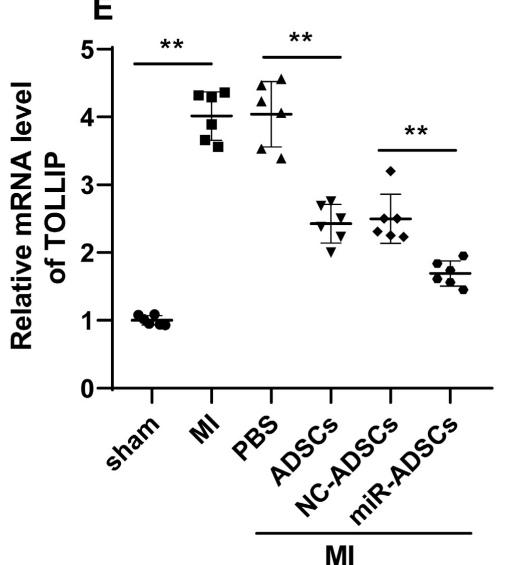

Fig. 4. miR-324-5p targets TOLLIP expression in myocardial tissues of $\mathrm{MI}$ rats. Note: $\mathrm{A}$, downstream target gene of miR-324-5p predicted by miRDB (http://mirdb.org/index.html) and Targetscan (http://www.targetscan. org/vert_71/) databases. B, binding site between miR-324-5p and TOLLIP was predicted by Targetscan database. $\mathrm{C}$, binding relation of miR-324$5 p$ and TOLLIP was verified through dual-luciferase reporter gene assay. $D$ and $E$, expressions of miR-324-5p (D) and TOLLIP (E) were measured by RT-qPCR. $N=6$, the cell experiment was repeated three times; the results in panel $\mathrm{C}$ were shown as mean \pm standard deviation. Two-way ANOVA was used to analyze data in panel C, and Sidak's multiple comparisons test was applied for post hoc test. One-way ANOVA was used to analyze data in panels $D$ and $E$, and Tukey's multiple comparisons test was applied for post hoc test. ${ }^{*} \mathrm{p}<0.01$. MI: myocardial infarction, NC-ADSCs: ADSCs after lentivirus infection with miR-324-5p negative control, miR-ADSCs: ADSCs after lentivirus infection with miR324-5p overexpression vectors. regulated TOLLIP expression; while miR-324-5p overexpression led to downregulated TOLLIP $(p<0.01$, Fig. $4 \mathrm{D}$ and $4 \mathrm{E})$. That's to say, miR-324-5p targeted TOLLIP.

\section{TOLLIP overexpression spoils the promotive role of miR-324-5p-modified ADSCs in post-MI repair in rats}

To confirm that miR-324-5p-modified ADSCs promoted myocardial repair by targeting TOLLIP in myocardial tissues, functional rescue assay was conducted as TOLLIP expression was successfully upregulated in myocardial tissues via the injection of Ad-TOLLIP in rats from the miR-ADSCs group $(p<0.01$, Fig. $5 \mathrm{~A})$, and it was subsequently uncovered that the myocardial function of $\mathrm{MI}$ rats was attenuated $(p<0.01$, Fig. $5 B)$, and the levels of cTnT and CK-MB in serum were elevated $(p<0.01$, Fig. 5C). Additionally, after overexpressing TOLLIP, both the degree of MI and apoptotic cells were greatly increased in the myocardial tissues of MI rats $(p<0.01$, Fig. $5 D$ and $5 \mathrm{E})$. These results illustrated that TOLLIP overexpression spoiled the promotive role of miR-324-5p-modified ADSCs in post-MI repair in rats.

\section{Discussion}

MI referred to a concept in which plaques accumulated at interior wall of arteries to block blood flow toward heart and lead to oxidative stress-induced myocardial injuries, eventually causing cardiac disease (2). For the properties of availability and renewability, ADSCs emerged as a popular choice for target treatment of post-MI repair (35). Accumulating reports suggested that miRs-modified ADSC therapy helped to reduce fibrosis, inflammation and cell death in MI when compared with ADSC therapy alone (17). Furthermore, miR dysregulation was critical player in myocardial hypertrophy and cardiac fatigue of MI (36). Woods and his colleagues uncovered that miR-324-5p could positively regulate MSC osteogenic development to maintain homeostasis and embryogenesis in patients with osteoarthritis (37). Therefore, we were inspired to explore the possible role of miR-324-5p-modified ADSCs in post-MI repair.

Firstly, in our experiments, the fact that miR-324-5p promoted ADSC proliferation and migration was noticed. It was recently unveiled that different miRs were related 

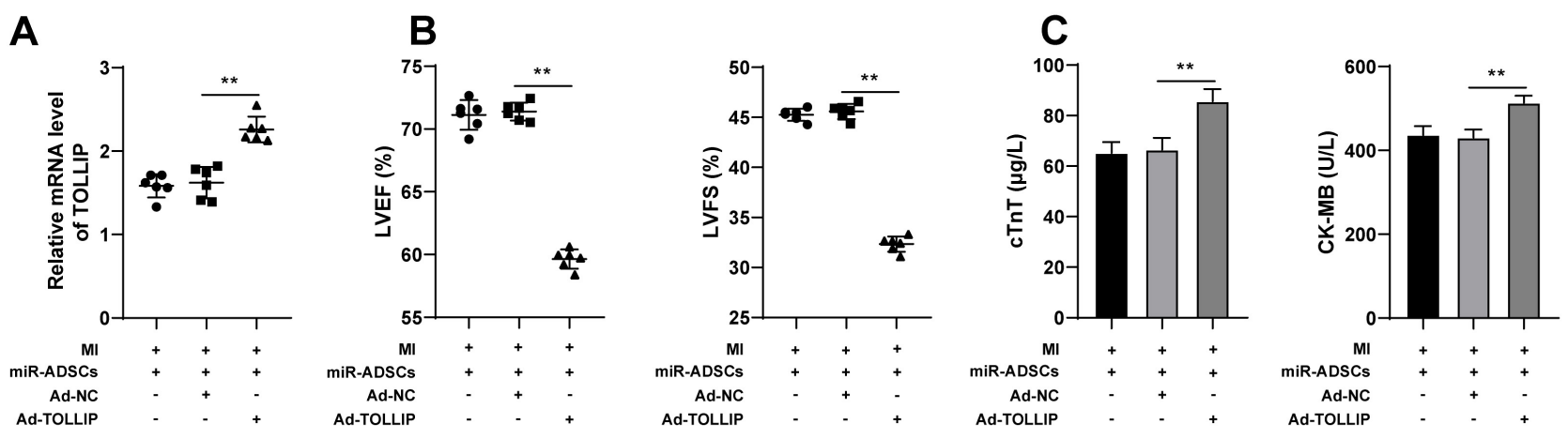

D

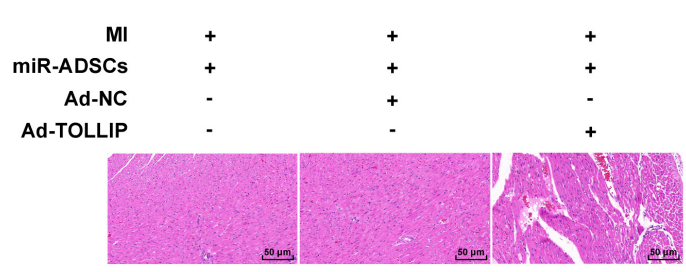

E

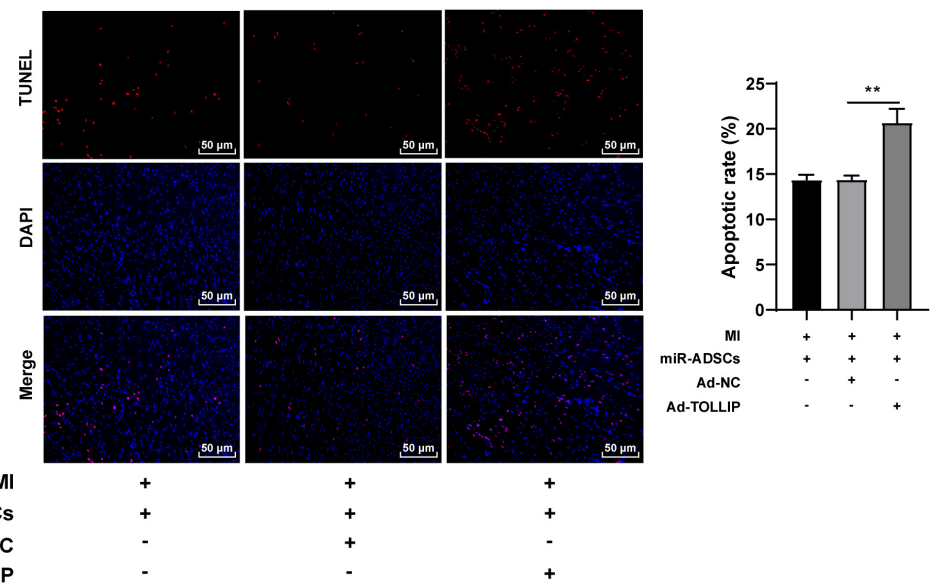

Fig. 5. TOLLIP overexpression spoils the promotive role of miR-324-5p-modified ADSCs in post-Ml repair in rats. Note: Ad-TOLLIP was injected in rats from the miR-ADSCs group, with Ad-NC as control. A, silencing efficiency of Ad-TOLLIP was verified by RT-qPCR. B, 28 days after ADSCs transplantation, myocardial function of rats were tested by echocardiography, $\mathrm{N}=12$. C, levels of cTnT and CK-MB in serum of rats were measured, $\mathrm{N}=12$. $\mathrm{D}, \mathrm{Ml}$ degree in rats was observed by $\mathrm{HE}$ staining. $\mathrm{E}$, apoptosis in $\mathrm{Ml}$ rats was verified through TUNEL staining. $N=6$, the results of panels $C$ and $E$ were shown as mean \pm standard deviation. One-way ANOVA was used to analyze data in panels A, B, C and E. Tukey's multiple comparisons test was applied for post hoc test. ${ }^{* *} \mathrm{p}<0.01$. Ml: myocardial infarction, NC-ADSCs: ADSCs after lentivirus infection with miR-324-5p negative control, miR-ADSCs: ADSCs after lentivirus infection with miR-324-5p overexpression vectors, Ad-TOLLIP: TOLLIP adenovirus.

to the biological processes of SCs including dissemination, differentiation and apoptosis $(38,39)$. Interestingly, miR324-3p propelled keratinocyte expansion, differentiation and self-renewal (40). On the other hand, inhibition of miR-324-5p impeded synapse construction of astrocyte neurons, which might incur senescence, inflammatory response and neurodegenerative disorders (41). Furthermore, miR-324-3p was activated in adipose tissues at multiple organs of obese mice, and downregulation of miR-324-3p resulted in loss of fat and body-weight, suggesting the positive correlation of miR-324-3p and ADSCs (42). Meanwhile, the interaction between miRs and ADSCs has gained a lot of success. For instance, encouraged by miR-375, ADSCs could better strengthen bone regeneration (43). Besides, miR-181-5p-modified ADSCs restored injured liver cells and interrupt liver fibrosis (44). From these progresses mentioned above, the positive crosstalk of miR-324-3p and ADSCs could be a contributory factor in post-MI repair. Consequently, we found that miR-324-5p-modified ADSCs enhanced post-MI repair in rats with the involvement of decreased levels of cTnT and CK-MB and compromised apoptosis. Apoptosis brought about retarded heart function and severe heart damage (45). CK-MB and cTnT, as common targets in heart diseases, were correlated with many cardiac specific miRs to regulate acute coronary syndrome (46). miR-324-5p overexpression could efficiently alleviate angiogenesis and pernicious cell expansion, eventually decelerating pulmonary arterial hypertension (47). On the other hand, miR-324 relieved cardiomyocyte damage and oxidative stress of myocardial injury triggered by hypoxia/reoxygenation (13). Surprisingly, Huang and his colleagues (48) reported that miR-324-5p activation atte- 
nuated apoptosis and ventricular cardiomyocyte injury exacerbated by myocardial ischemia-reperfusion to relieve MI. Moreover, miR-modified MSCs maintained ventricular construction and expedited post-MI repair through depleting cell death and apoptosis (49). As a class of MSCs, ADSC transplantation was extensively applied in alleviation and repair of diseases like diabetes, facial restoration, osteoarthritis and cardiac disorders (50). The accumulation of ADSCs after MI unleashed the release of vascular endothelial growth factor and led to angiogenesis (51). Moreover, ADSCs were able to remodel blood vessels when they differentiated to vascular net (52). Significantly, it was previously unmasked that miR-342-5p modified ADSCs potentiated endothelial cells to defense atherosclerosis (53). In addition to this, ADSCs limited the level of cTnT (54). Altogether, it might be quite certain that miR-342-5p modified ADSCs served as contributor to post-MI repair.

Last but not least, we found that miR-324-5p targeted TOLLIP in myocardial tissues of MI rats. As a predominant cytokine in immunological response guided by Toll-like receptor, TOLLIP proactively participated autophagy and inflammation in tumors and human disorders related to liver, neurodegeneration and angiocarpy $(55,56)$. According to Ryan et al. (57) TOLLIP upregulation was coupled with growing expression of Parkin and it represented a manifestation of mitochondrial injury, which finally resulted in deteriorative Parkinson's disease. A recent finding revealed that TOLLIP exhaustion plainly degraded liver damage inflicted by ischemia-reperfusion (58). That's to say, TOLLIP imposed adverse effect on diseases related to inflammatory reaction and immune system. Consistently, our experiment uncovered that TOLLIP overexpression spoiled the promotive role of miR-324-5p-modified ADSCs in post-MI repair in rats as presented by elevated levels of levels of cTnT and CK-MB and improved apoptosis. As a pivotal diagnosis biomarker in MI, cTnT upregulation could greatly destroy heart function (59). TOLLIP depletion was associated with stable plaques of atherosclerosis areas, attenuated inflammatory response, growing circulating lipids and impaired lipophagy in atherosclerosis (60). Besides, as an acknowledged cardiovascular modulator, TOLLIP aggravated neurocyte apoptosis and inflammatory reaction in individuals injured by ischemia-reperfusion (61). All in all, miR-342-5p modified ADSCs could work as a promising inhibitor of MI by targeting TOLLIP.

In summary, our findings revealed that miR-342-5p modified ADSCs promoted post-MI repair by targeting TOLLIP. These results originally discovered an approach for MI treatment. Still, some limitations are to be solved. For example, this study merely uncovered the modifying effect of miR-324-5p on ADSCs, without clarifying it there is any effect on SCs. In addition, whether there are other miRNAs involved is yet to know. Furthermore, other downstream target genes of miR-324-5p need to be investigated. The purpose of this study is to investigate the role of miRNA modified ADSCs in myocardial repair after MI. We did not detect ADSCs differentiation in rat hearts, but some studies showed that the adipose stem cells of heart transplantation were differentiated into cardiomyocytes as a whole $(62,63)$, which we will take as our next research direction. The "paracrine effect" has been proposed as a promising strategy to boost endogenous reparative and regenerative mechanisms within the cardiac tissue (64). Stem cells are known to secrete various factors that have paracrine activities; they home to hypoxic and/or inflamed areas, and release trophic factors that hasten endogenous repair and the secreted bioactive factors limit local immune system, enhance angiogenesis, inhibit fibrosis and apoptosis, and stimulate differentiation of tissue-residing stem cells (65). Once transplanted into a damaged organ, MSCs release paracrine factors that nurture the injured area, prevent further adverse cardiac remodeling, and mediate tissue repair along with vasculature (66-68). We will focus on whether miR-324-5p is carried into cardiomyocytes by ADSCs through paracrine effect in the next research.

\section{Acknowledgments}

This research was supported by funds from the Natural Science Foundation of Liaoning Province (2019-MS-144).

\section{Potential Conflict of Interest}

The authors have no conflicting financial interest.

\section{Ethical Approval and Consent to Participate}

This study was granted and oversaw by the ethics committee of The Third Affiliated Hospital of Jinzhou Medical University. Significant efforts were made to minimize both the number of animals used and their suffering.

\section{Author Contributions}

Zhou Ji: study concepts, literature research, data analysis, experimental studies, manuscript writing and review; Chan Wang: study design, literature research, experimental studies and manuscript editing; Qing Tong: definition of intellectual content, data acquisition and statistical analysis. 


\section{References}

1. Ong SB, Hernández-Reséndiz S, Crespo-Avilan GE, Mukhametshina RT, Kwek XY, Cabrera-Fuentes HA, Hausenloy DJ. Inflammation following acute myocardial infarction: multiple players, dynamic roles, and novel therapeutic opportunities. Pharmacol Ther 2018;186:73-87

2. Lu L, Liu M, Sun R, Zheng Y, Zhang P. Myocardial infarction: symptoms and treatments. Cell Biochem Biophys 2015;72:865-867

3. Lisowska A, Makarewicz-Wujec M, Filipiak KJ. Risk factors, prognosis, and secondary prevention of myocardial infarction in young adults in Poland. Kardiol Pol 2016;74: 1148-1153

4. Fang L, Moore XL, Dart AM, Wang LM. Systemic inflammatory response following acute myocardial infarction. J Geriatr Cardiol 2015;12:305-312

5. Thakker R, Yang P. Mesenchymal stem cell therapy for cardiac repair. Curr Treat Options Cardiovasc Med 2014; $16: 323$

6. Bagno L, Hatzistergos KE, Balkan W, Hare JM. Mesenchymal stem cell-based therapy for cardiovascular disease: progress and challenges. Mol Ther 2018;26:1610-1623

7. Kim JH, Joo HJ, Kim M, Choi SC, Lee JI, Hong SJ, Lim DS. Transplantation of adipose-derived stem cell sheet attenuates adverse cardiac remodeling in acute myocardial infarction. Tissue Eng Part A 2017;23:1-11

8. Otto Beitnes J, Oie E, Shahdadfar A, Karlsen T, Müller RM, Aakhus S, Reinholt FP, Brinchmann JE. Intramyocardial injections of human mesenchymal stem cells following acute myocardial infarction modulate scar formation and improve left ventricular function. Cell Transplant 2012;21: 1697-1709

9. Peng C, Pei H, Wei F, Tian X, Deng J, Yan C, Li Y, Sun M, Zhang J, Liu D, Rong J, Wang J, Gao E, Li S, Han Y. Cellular repressor of E1A-stimulated gene overexpression in bone mesenchymal stem cells protects against rat myocardial infarction. Int J Cardiol 2015;183:232-241

10. Xu J, Huang Z, Lin L, Fu M, Gao Y, Shen Y, Zou Y, Sun A, Qian J, Ge J. miR-210 over-expression enhances mesenchymal stem cell survival in an oxidative stress environment through antioxidation and c-Met pathway activation. Sci China Life Sci 2014;57:989-997

11. Ham O, Lee SY, Lee CY, Park JH, Lee J, Seo HH, Cha MJ, Choi E, Kim S, Hwang KC. let-7b suppresses apoptosis and autophagy of human mesenchymal stem cells transplanted into ischemia/reperfusion injured heart 7by targeting caspase-3. Stem Cell Res Ther 2015;6:147

12. Fiedler J, Thum T. MicroRNAs in myocardial infarction. Arterioscler Thromb Vasc Biol 2013;33:201-205

13. Han X, Chen X, Han J, Zhong Y, Li Q, An Y. MiR-324/ SOCS3 axis protects against hypoxia/reoxygenation-induced cardiomyocyte injury and regulates myocardial ischemia via $\mathrm{TNF} / \mathrm{NF}-\kappa \mathrm{B}$ signaling pathway. Int Heart J 2020;61:1258-1269

14. Zhou X, Shi X, Wang J, Zhang X, Xu Y, Liu Y, Li X, Yang
G. miR-324-5p promotes adipocyte differentiation and lipid droplet accumulation by targeting Krueppel-like factor 3 (KLF3). J Cell Physiol 2020;235:7484-7495

15. Chen P, Zhong J, Ye J, He Y, Liang Z, Cheng Y, Zheng J, Chen H, Chen C. miR-324-5p protects against oxidative stress-induced endothelial progenitor cell injury by targeting Mtfr1. J Cell Physiol 2019;234:22082-22092

16. Wan N, Liu X, Zhang XJ, Zhao Y, Hu G, Wan F, Zhang $\mathrm{R}, \mathrm{Zhu} \mathrm{X}$, Xia H, Li H. Toll-interacting protein contributes to mortality following myocardial infarction through promoting inflammation and apoptosis. Br J Pharmacol 2015; 172:3383-3396

17. Pan J, Alimujiang M, Chen Q, Shi H, Luo X. Exosomes derived from miR-146a-modified adipose-derived stem cells attenuate acute myocardial infarction-induced myocardial damage via downregulation of early growth response factor 1. J Cell Biochem 2019;120:4433-4443

18. Zeng YL, Zheng $\mathrm{H}$, Chen $\mathrm{QR}$, Yuan $\mathrm{XH}$, Ren JH, Luo XF, Chen P, Lin ZY, Chen SZ, Wu XQ, Xiao M, Chen YQ, Chen ZZ, Hu JD, Yang T. Bone marrow-derived mesenchymal stem cells overexpressing MiR-21 efficiently repair myocardial damage in rats. Oncotarget 2017;8:29161-29173

19. Li M, Ke QF, Tao SC, Guo SC, Rui BY, Guo YP. Fabrication of hydroxyapatite/chitosan composite hydrogels loaded with exosomes derived from miR-126-3p overexpressed synovial mesenchymal stem cells for diabetic chronic wound healing. J Mater Chem B 2016;4:6830-6841

20. Chen Y, Zhao Y, Chen W, Xie L, Zhao ZA, Yang J, Chen Y, Lei W, Shen Z. MicroRNA-133 overexpression promotes the therapeutic efficacy of mesenchymal stem cells on acute myocardial infarction. Stem Cell Res Ther 2017;8:268

21. Rao Z, Shen D, Chen J, Jin L, Wu X, Chen M, Li L, Chu $\mathrm{M}$, Lin J. Basic fibroblast growth factor attenuates injury in myocardial infarction by enhancing hypoxia-inducible factor-1 alpha accumulation. Front Pharmacol 2020;11:1193

22. Peng Y, Zhao JL, Peng ZY, Xu WF, Yu GL. Exosomal miR-25-3p from mesenchymal stem cells alleviates myocardial infarction by targeting pro-apoptotic proteins and EZH2. Cell Death Dis 2020;11:317

23. Liu J, Jiang M, Deng S, Lu J, Huang H, Zhang Y, Gong $\mathrm{P}$, Shen $\mathrm{X}$, Ruan H, Jin $M$, Wang H. miR-93-5p-containing exosomes treatment attenuates acute myocardial infarction-induced myocardial damage. Mol Ther Nucleic Acids 2018;11:103-115

24. Wu Q, Wang J, Tan WLW, Jiang Y, Wang S, Li Q, Yu X, Tan J, Liu S, Zhang P, Tiang Z, Chen Z, Foo RS, Yang HT. Extracellular vesicles from human embryonic stem cell-derived cardiovascular progenitor cells promote cardiac infarct healing through reducing cardiomyocyte death and promoting angiogenesis. Cell Death Dis 2020;11:354

25. Sun L, Zhu W, Zhao P, Wang Q, Fan B, Zhu Y, Lu Y, Chen Q, Zhang J, Zhang F. Long noncoding RNA UCAl from hypoxia-conditioned hMSC-derived exosomes: a novel molecular target for cardioprotection through miR-873-5p/ XIAP axis. Cell Death Dis 2020;11:696

26. Luo Q, Guo D, Liu G, Chen G, Hang M, Jin M. Exosomes 
from MiR-126-overexpressing Adscs are therapeutic in relieving acute myocardial ischaemic injury. Cell Physiol Biochem 2017;44:2105-2116

27. Huang L, Yang L, Ding Y, Jiang X, Xia Z, You Z. Human umbilical cord mesenchymal stem cells-derived exosomes transfers microRNA-19a to protect cardiomyocytes from acute myocardial infarction by targeting SOX6. Cell Cycle 2020;19:339-353

28. Xu H, Wang Z, Liu L, Zhang B, Li B. Exosomes derived from adipose tissue, bone marrow, and umbilical cord blood for cardioprotection after myocardial infarction. J Cell Biochem 2020;121:2089-2102

29. Liang YP, Liu Q, Xu GH, Zhang J, Chen Y, Hua FZ, Deng CQ, Hu YH. The lncRNA ROR/miR-124-3p/TRAF6 axis regulated the ischaemia reperfusion injury-induced inflammatory response in human cardiac myocytes. J Bioenerg Biomembr 2019;51:381-392

30. Livak KJ, Schmittgen TD. Analysis of relative gene expression data using real-time quantitative PCR and the 2(-Delta Delta C(T)) Method. Methods 2001;25:402-408

31. Yang WJ, Yang DD, Na S, Sandusky GE, Zhang Q, Zhao G. Dicer is required for embryonic angiogenesis during mouse development. J Biol Chem 2005;280:9330-9335

32. Amado LC, Saliaris AP, Schuleri KH, St John M, Xie JS, Cattaneo S, Durand DJ, Fitton T, Kuang JQ, Stewart G, Lehrke S, Baumgartner WW, Martin BJ, Heldman AW, Hare JM. Cardiac repair with intramyocardial injection of allogeneic mesenchymal stem cells after myocardial infarction. Proc Natl Acad Sci U S A 2005;102:11474-11479

33. Zhang GW, Gu TX, Guan XY, Sun XJ, Qi X, Li XY, Wang $\mathrm{XB}, \mathrm{Lv} \mathrm{F}, \mathrm{Yu} \mathrm{L}$, Jiang DQ, Tang R. HGF and IGF-1 promote protective effects of allogeneic BMSC transplantation in rabbit model of acute myocardial infarction. Cell Prolif 2015;48:661-670

34. Yu J, Li M, Qu Z, Yan D, Li D, Ruan Q. SDF-1/CXCR4mediated migration of transplanted bone marrow stromal cells toward areas of heart myocardial infarction through activation of PI3K/Akt. J Cardiovasc Pharmacol 2010;55: 496-505

35. Mazo M, Gavira JJ, Pelacho B, Prosper F. Adipose-derived stem cells for myocardial infarction. J Cardiovasc Transl Res 2011;4:145-153

36. van Rooij E, Sutherland LB, Thatcher JE, DiMaio JM, Naseem RH, Marshall WS, Hill JA, Olson EN. Dysregulation of microRNAs after myocardial infarction reveals a role of miR-29 in cardiac fibrosis. Proc Natl Acad Sci U S A 2008;105:13027-13032

37. Woods S, Barter MJ, Elliott HR, McGillivray CM, Birch MA, Clark IM, Young DA. miR-324-5p is up regulated in end-stage osteoarthritis and regulates Indian Hedgehog signalling by differing mechanisms in human and mouse. Matrix Biol 2019;77:87-100

38. Shi M, Ma X, Yang Q, Wang W, Li X, Song X, Li Y, Xie Y, Dang Y. miR-362-3p targets orosomucoid 1 to promote cell proliferation, restrain cell apoptosis and thereby mitigate hypoxia/reoxygenation-induced cardiomyocytes injury.
Cardiovasc Toxicol 2021;21:387-398

39. Mao Y, Hou B, Shan L, Sun X, Wang L. Aberrantly upregulated miR-142-3p inhibited the proliferation and invasion of trophoblast cells by regulating FOXM1. Placenta 2021;104:253-260

40. Mohammadi P, Nilforoushzadeh MA, Youssef KK, SharifiZarchi A, Moradi S, Khosravani P, Aghdami R, Taheri P, Hosseini Salekdeh G, Baharvand H, Aghdami N. Defining microRNA signatures of hair follicular stem and progenitor cells in healthy and androgenic alopecia patients. J Dermatol Sci 2021;101:49-57

41. Sun C, Zhu L, Ma R, Ren J, Wang J, Gao S, Yang D, Ning $\mathrm{K}$, Ling B, Lu B, Chen X, Xu J. Astrocytic miR-324-5p is essential for synaptic formation by suppressing the secretion of CCL5 from astrocytes. Cell Death Dis 2019;10:141

42. Li D, Liu Y, Gao W, Han J, Yuan R, Zhang M, Pang W. Inhibition of miR-324-5p increases PM20D1-mediated white and brown adipose loss and reduces body weight in juvenile mice. Eur J Pharmacol 2019;863:172708

43. Chen S, Tang Y, Liu Y, Zhang P, Lv L, Zhang X, Jia L, Zhou Y. Exosomes derived from miR-375-overexpressing human adipose mesenchymal stem cells promote bone regeneration. Cell Prolif 2019;52:e12669

44. Qu Y, Zhang Q, Cai X, Li F, Ma Z, Xu M, Lu L. Exosomes derived from miR-181-5p-modified adipose-derived mesenchymal stem cells prevent liver fibrosis via autophagy activation. J Cell Mol Med 2017;21:2491-2502

45. Han F, Chen Q, Su J, Zheng A, Chen K, Sun S, Wu H, Jiang $\mathrm{L}$, Xu $\mathrm{X}$, Yang $\mathrm{M}$, Yang $\mathrm{F}$, Zhu J, Zhang $\mathrm{L}$. MicroRNA-124 regulates cardiomyocyte apoptosis and myocardial infarction through targeting Dhcr24. J Mol Cell Cardiol 2019;132:178-188

46. Mushtaque RS, Hameed S, Mushtaque R, Idrees M, Siraj F. Role of cardio-specific micro-ribonucleic acids and correlation with cardiac biomarkers in acute coronary syndrome: a comprehensive systematic review. Cureus 2019;11: e5878

47. Sindi HA, Russomanno G, Satta S, Abdul-Salam VB, Jo KB, Qazi-Chaudhry B, Ainscough AJ, Szulcek R, Jan Bogaard H, Morgan CC, Pullamsetti SS, Alzaydi MM, Rhodes CJ, Piva R, Eichstaedt CA, Grünig E, Wilkins MR, Wojciak-Stothard B. Therapeutic potential of KLF2-induced exosomal microRNAs in pulmonary hypertension. Nat Commun 2020;11:1185

48. Huang L, Guo B, Liu S, Miao C, Li Y. Inhibition of the LncRNA Gpr19 attenuates ischemia-reperfusion injury after acute myocardial infarction by inhibiting apoptosis and oxidative stress via the miR-324-5p/Mtfrl axis. IUBMB Life 2020;72:373-383

49. Cheng J, Zhang P, Jiang H. Let-7b-mediated pro-survival of transplanted mesenchymal stem cells for cardiac regeneration. Stem Cell Res Ther 2015;6:216

50. Minteer D, Marra KG, Rubin JP. Adipose-derived mesenchymal stem cells: biology and potential applications. Adv Biochem Eng Biotechnol 2013;129:59-71

51. Gao L, Mei S, Zhang S, Qin Q, Li H, Liao Y, Fan H, Liu 
Z, Zhu H. Cardio-renal exosomes in myocardial infarction serum regulate proangiogenic paracrine signaling in adipose mesenchymal stem cells. Theranostics 2020;10:10601073

52. Ni H, Zhao Y, Ji Y, Shen J, Xiang M, Xie Y. Adipose-derived stem cells contribute to cardiovascular remodeling. Aging (Albany NY) 2019;11:11756-11769

53. Xing $\mathrm{X}, \mathrm{Li}$ Z, Yang $\mathrm{X}$, Li M, Liu C, Pang Y, Zhang L, Li X, Liu G, Xiao Y. Adipose-derived mesenchymal stem cells-derived exosome-mediated microRNA-342-5p protects endothelial cells against atherosclerosis. Aging (Albany NY) 2020;12:3880-3898

54. Ibarra-Ibarra BR, Franco M, Paez A, López EV, Massó F. Improved efficiency of cardiomyocyte-like cell differentiation from rat adipose tissue-derived mesenchymal stem cells with a directed differentiation protocol. Stem Cells Int 2019;2019:8940365

55. Li X, Goobie GC, Zhang Y. Toll-interacting protein impacts on inflammation, autophagy, and vacuole trafficking in human disease. J Mol Med (Berl) 2021;99:21-31

56. Capelluto DG. Tollip: a multitasking protein in innate immunity and protein trafficking. Microbes Infect 2012;14: 140-147

57. Ryan TA, Phillips EO, Collier CL, Jb Robinson A, Routledge D, Wood RE, Assar EA, Tumbarello DA. Tollip coordinates Parkin-dependent trafficking of mitochondrial-derived vesicles. EMBO J 2020;39:e102539

58. Yan ZZ, Huang YP, Wang X, Wang HP, Ren F, Tian RF, Cheng X, Cai J, Zhang Y, Zhu XY, She ZG, Zhang XJ, Huang Z, Li H. Integrated omics reveals Tollip as an regulator and therapeutic target for hepatic ischemia-reperfusion injury in mice. Hepatology 2019;70:1750-1769

59. Wang Y, Qi X, Wang C, Zhao D, Wang H, Zhang J. Effects of propofol on myocardial ischemia-reperfusion injury in rats with type-2 diabetes mellitus. Biomed Rep 2017;6:69-74

60. Chen K, Yuan R, Zhang Y, Geng S, Li L. Tollip deficiency alters atherosclerosis and steatosis by disrupting lipophagy. J Am Heart Assoc 2017;6:e004078

61. Li M, Feng B, Wang L, Guo S, Zhang P, Gong J, Zhang $\mathrm{Y}$, Zheng A, Li H. Tollip is a critical mediator of cerebral ischaemia-reperfusion injury. J Pathol 2015;237:249-262

62. Zhu Y, Liu T, Song K, Fan X, Ma X, Cui Z. Adipose-derived stem cell: a better stem cell than BMSC. Cell Biochem Funct 2008;26:664-675

63. Meliga E, Strem BM, Duckers HJ, Serruys PW. Adiposederived cells. Cell Transplant 2007;16:963-970

64. Balbi C, Costa A, Barile L, Bollini S. Message in a bottle: upgrading cardiac repair into rejuvenation. Cells 2020;9: 724

65. Sondergaard C, Hess D, Rosova I, Hohm S, Wirthlin L, Lahey R, Lindsey M, Walker J, Bauer G, Zhou P, Nolta J. Adult human stem cells exert therapeutic effects to repair damaged tissues in xenograft systems through secretion of trophic factors rather than direct incorporation and expansion. Blood 2007;110:3693

66. Zhang N, Zhu J, Ma Q, Zhao Y, Wang Y, Hu X, Chen J, Zhu W, Han Z, Yu H. Exosomes derived from human umbilical cord MSCs rejuvenate aged MSCs and enhance their functions for myocardial repair. Stem Cell Res Ther 2020; $11: 273$

67. Sid-Otmane C, Perrault LP, Ly HQ. Mesenchymal stem cell mediates cardiac repair through autocrine, paracrine and endocrine axes. J Transl Med 2020;18:336

68. Bui TVA, Hwang JW, Lee JH, Park HJ, Ban K. Challenges and limitations of strategies to promote therapeutic potential of human mesenchymal stem cells for cell-based cardiac repair. Korean Circ J 2021;51:97-113 